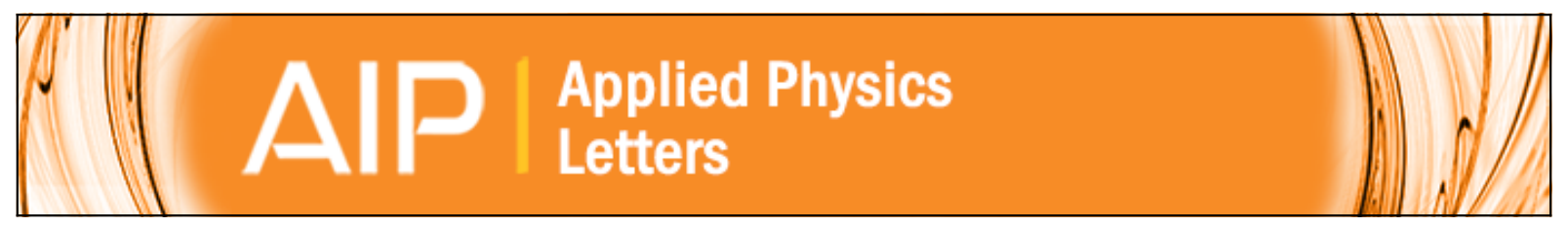

\title{
Amplified spontaneous emission and efficient tunable laser emission from a substituted thiophene-based oligomer
}

Dario Pisignano, Marco Anni, Giuseppe Gigli, Roberto Cingolani, Margherita Zavelani-Rossi, Guglielmo Lanzani, Giovanna Barbarella, and Laura Favaretto

Citation: Applied Physics Letters 81, 3534 (2002); doi: 10.1063/1.1519735

View online: http://dx.doi.org/10.1063/1.1519735

View Table of Contents: http://scitation.aip.org/content/aip/journal/apl/81/19?ver=pdfcov

Published by the AIP Publishing

\section{Articles you may be interested in}

Amplified spontaneous emission from cyano substituted oligo( $p$-phenylene vinylene) single crystal with very high photoluminescent efficiency

Appl. Phys. Lett. 90, 141110 (2007); 10.1063/1.2720298

Origin of the amplified spontaneous emission from thiophene/phenylene co-oligomer single crystals: Towards cooligomer lasers

J. Appl. Phys. 99, 013518 (2006); 10.1063/1.2159083

Amplified spontaneous emission from a conjugated polymer undergone a high-temperature lithography cycle Appl. Phys. Lett. 86, 261104 (2005); 10.1063/1.1946189

Amplified spontaneous emission from a soluble thiophene-based oligomer

Appl. Phys. Lett. 78, 2679 (2001); 10.1063/1.1369392

Amplified spontaneous emission and lasing in conducting polymers and fluorescent dyes in opals as photonic crystals

Appl. Phys. Lett. 74, 2590 (1999); 10.1063/1.123907

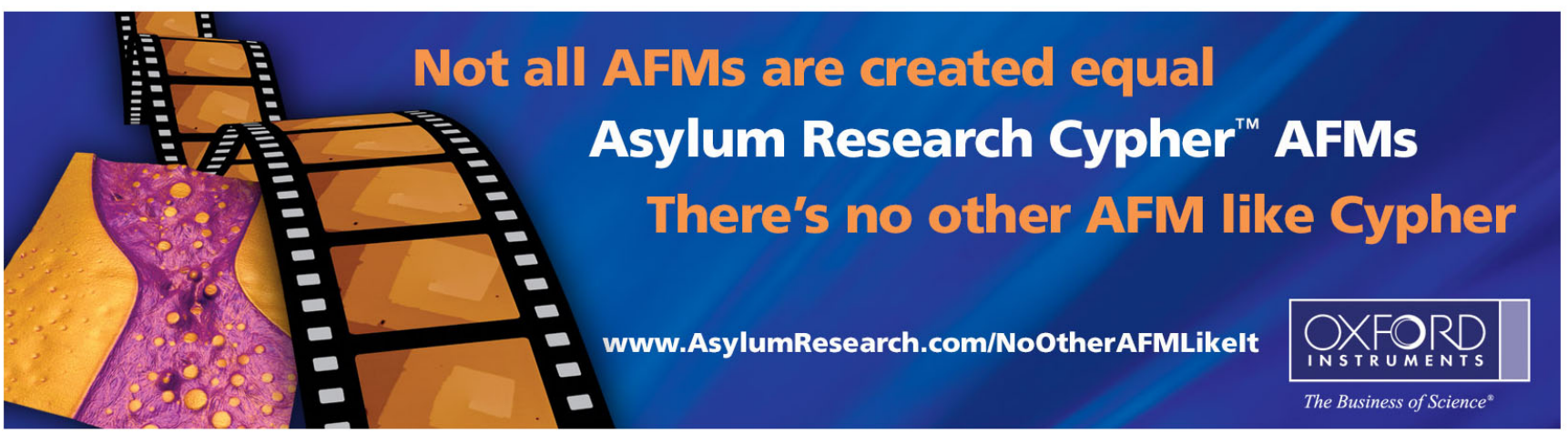




\title{
Amplified spontaneous emission and efficient tunable laser emission from a substituted thiophene-based oligomer
}

\author{
Dario Pisignano, ${ }^{\text {a) }}$ Marco Anni, Giuseppe Gigli, and Roberto Cingolani \\ NNL, National Nanotechnology Laboratory of Istituto Nazionale di Fisica della Materia (INFM), \\ clo Dipartimento di Ingegneria dell'Innovazione, via Arnesano, I-73100 Lecce, Italy \\ Margherita Zavelani-Rossi and Guglielmo Lanzani \\ INFM, CEQSE-CNR, Dipartimento di Fisica, Politecnico di Milano, Piazza Leonardo Da Vinci 32, \\ I-20133 Milano, Italy \\ Giovanna Barbarella and Laura Favaretto \\ Consiglio Nazionale delle Ricerche (CNR), ICOCEA, Area della Ricerca di Bologna, via Gobetti 101, \\ I-40129 Bologna, Italy
}

(Received 15 July 2002; accepted 14 September 2002)

\begin{abstract}
We investigated gain and lasing in spin-coated films of a soluble substituted oligothiophene. With increasing excitation power, the photoluminescence spectra show a clear line narrowing due to amplified spontaneous emission. We measure a low threshold $\left(20 \mu \mathrm{J} \mathrm{cm}^{-2}\right)$ for line narrowing and a large gain cross section $\left(6 \times 10^{-16} \mathrm{~cm}^{2}\right)$, indicating that this molecule is a promising active material for organic solid-state lasers. As a demonstrator, we realize a transverse electromagnetic $\left(\mathrm{TEM}_{00}\right)$ single-mode laser with tunable emission from the yellow to the red (a range of $37 \mathrm{~nm}$ ), with a pump threshold as low as $18 \mu \mathrm{J} \mathrm{cm}^{-2}$ and efficiency of $1.9 \%$. These results are among the best so far reported for organic lasers. (c) 2002 American Institute of Physics.
\end{abstract}

[DOI: $10.1063 / 1.1519735]$

Light-emitting organic materials, both conjugated polymers and low-molar-mass compounds, have several appealing characteristics for the realization of active optical devices: namely, high photoluminescence (PL) efficiency, wide tunability of the emission wavelength, as well as low cost and simple deposition techniques. Recently, the observation of optical gain from a wide range of organic compounds, both in solution ${ }^{1}$ and in thin films, ${ }^{2}$ further raised the interest into organic semiconductor lasers. A number of conjugated compounds have been demonstrated to show optical gain, ${ }^{3-7}$ and optically pumped lasing with different cavity geometries. $^{8-13}$ Among these compounds, functionalized thiophene-based oligomers are quite interesting, because of their wide color tunability, ${ }^{14,15}$ good chemical stability, ${ }^{16}$ high solubility in common organic solvents, and very good film-forming capability. In addition, the two main drawbacks of unsubstituted oligothiophenes, namely their low PL efficiency in the solid phase, $\eta_{\mathrm{PL}}$, and low electron affinity, $\chi$, have been completely overcome by the dioxide functionalization of the thiophene heteroatom, which allowed us to achieve values of $\eta_{\mathrm{PL}}$ up to $70 \%$ and of $\chi$ of about $3 \mathrm{~V} .{ }^{14}$ These features have been exploited to fabricate multicolor organic light-emitting diodes, with low turn-on voltage and high electroluminescence efficiency ${ }^{15}$ Moreover, optical gain with a cross section of $4 \times 10^{-18} \mathrm{~cm}^{2}$ and lasing with a threshold of $5 \mathrm{~mJ} \mathrm{~cm}^{-2}$ have been recently demonstrated in the substituted quinque-thiophene, $3,3^{\prime}, 4^{\prime \prime \prime}, 3^{\prime \prime \prime}$ Tetramethyl-3", $4^{\prime \prime}$ - dihexyl- $2,2^{\prime}: 5^{\prime}, 2^{\prime \prime}: 5^{\prime \prime}, 2^{\prime \prime \prime}: 5^{\prime \prime \prime}, 2^{\prime \prime \prime \prime}$ : quinquethiophene- $1^{\prime \prime}, 1^{\prime \prime}$-dioxide. ${ }^{17,18}$

In this letter, we demonstrate that a proper molecular engineering allows one to strongly improve the gain and las-

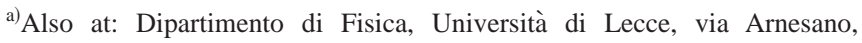
I-73100 Lecce, Italy; electronic mail: dario.pisignano@unile.it
}

ing capability. In particular, we studied the optical gain of spin coated films of $3,3^{\prime}, 4^{\prime \prime \prime}, 3^{\prime \prime \prime \prime}$ - Tetracyclehexyl-

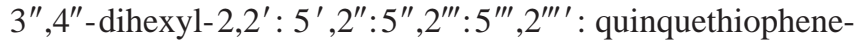
$1^{\prime \prime}, 1^{\prime \prime}$-dioxide compound [(T5oCx) structure in Fig. 1], showing very good electroluminescence yield. ${ }^{15}$ Intensity dependent PL experiments have revealed line narrowing, due to amplified spontaneous emission (ASE), with a threshold of $20 \mu \mathrm{J} \mathrm{cm}^{-2}$. The gain cross section determined by pumpprobe measurements was found to be $6 \times 10^{-16} \mathrm{~cm}^{-2}$, which is among the highest values reported for organic compounds. These characteristics have been exploited to fabricate a solidstate laser using a neat film in a plane-concave resonator. The laser exhibits single-mode transverse electromagnetic $\left(\mathrm{TEM}_{00}\right)$ emission, tunable from the yellow to the red, on a $37 \mathrm{~nm}$ wide wavelength range centered at $594 \mathrm{~nm}$, with a pump threshold as low as $18 \mu \mathrm{J} \mathrm{cm}^{-2}$, and efficiency up to $1.9 \%$.

$\mathrm{T} 5 \mathrm{oCx}$ films were cast by spin coating on Corning glass substrates, from chloroform solutions (concentration $\cong 7$ $\times 10^{-2} \mathrm{M}$ ), stirred for $2 \mathrm{~h}$ at room temperature. A spincoating speed of $1600 \mathrm{rpm}$ was used to obtain neat films of thickness about $440 \mathrm{~nm}$, measured by a profilometer. The second harmonic $(390 \mathrm{~nm})$ of a Kerr lens mode-locked Ti-

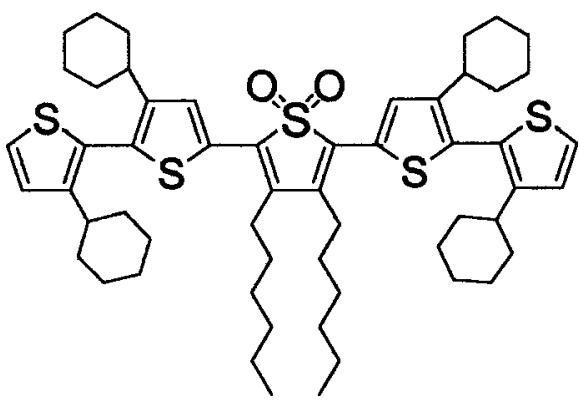

FIG. 1. Molecular structure of T5oCx. 


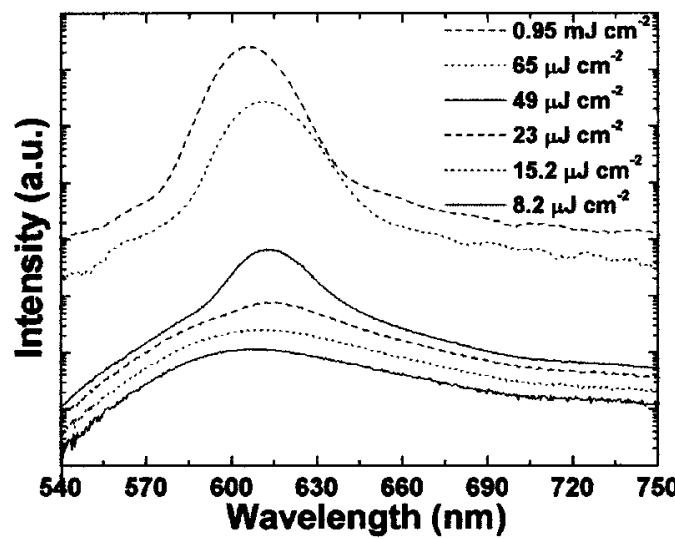

FIG. 2. Emission spectra collected at right angle for different absorbed excitation fluences.

:Sapphire laser with chirped-pulse amplification, delivering $150 \mathrm{fs}, 650 \mu \mathrm{J}$ pulses at $780 \mathrm{~nm}$ with a repetition rate of 1 $\mathrm{kHz}$ was used as the excitation source. The PL dependence on the pump excitation intensity was studied by focusing the pump beam on the sample by a cylindrical lens, thus obtaining a rectangular $100 \mu \mathrm{m} \times 7 \mathrm{~mm}$ excitation stripe. The luminescence emitted by the film was collected from the sample edge in a direction parallel to the film slab. Laser action was obtained by a plane-concave external resonator, consisting of a high reflectivity $(R)$ dielectric planar mirror ( $R \cong 99.5 \%$ in the gain spectral region of $\mathrm{T} 5 \mathrm{oCx}$ ), and a dielectric concave mirror, with a $8 \mathrm{~m}$ radius of curvature and $R \cong 98 \%$, acting as the output coupler. The active material was directly spin coated onto the high reflectivity mirror. Fine control of the cavity length was achieved by mounting the mirrors on high-precision translation stages. The laser was optically pumped longitudinally through the high reflector: $68 \%$ of the transmitted beam was absorbed by the film. The input and output energies of the laser were measured by a silicon optometer, and the emission spectra were recorded by an optical multichannel analyzer, with a resolution of 1.2 $\mathrm{nm}$. All measurements were carried out at room temperature and in air.

The PL spectra as a function of the excitation density are displayed in Fig. 2. At low absorbed excitation fluences, only a broad and featureless PL spectrum is visible, whereas for pump energies above $20 \mu \mathrm{J} \mathrm{cm}^{-2}$, a narrow peak appears at $615 \mathrm{~nm}$. We note that this value is more than one order of magnitude lower than that reported for sexithiophene single crystals $^{19}$ and almost 50 times lower than that of other functionalized oligothiophenes. ${ }^{17}$ The broad PL band and the narrow peak exhibit a different dependence on the excitation energy, as shown in Fig. 3. The broad PL background signal weakly grows in the investigated excitation range (empty dots in Fig. 3), whereas the total intensity, I, (squares in Fig. 3) due to the narrow band, shows an approximately exponential increase without a distinct threshold for pump densities up to about $0.2 \mathrm{~mJ} \mathrm{~cm}^{-2}$, and then saturates. ${ }^{20}$ This behavior agrees with ASE assisted by the asymmetric planar waveguiding due to the different refractive index of substrate, gain medium, and air.

In addition, we found that by increasing the excitation density, a redshift $(26 \mathrm{meV})$ of the peak energy occurs up to $0.02 \mathrm{~mJ} \mathrm{~cm}$, followed by a blueshift of $34 \mathrm{meV}$ for higher

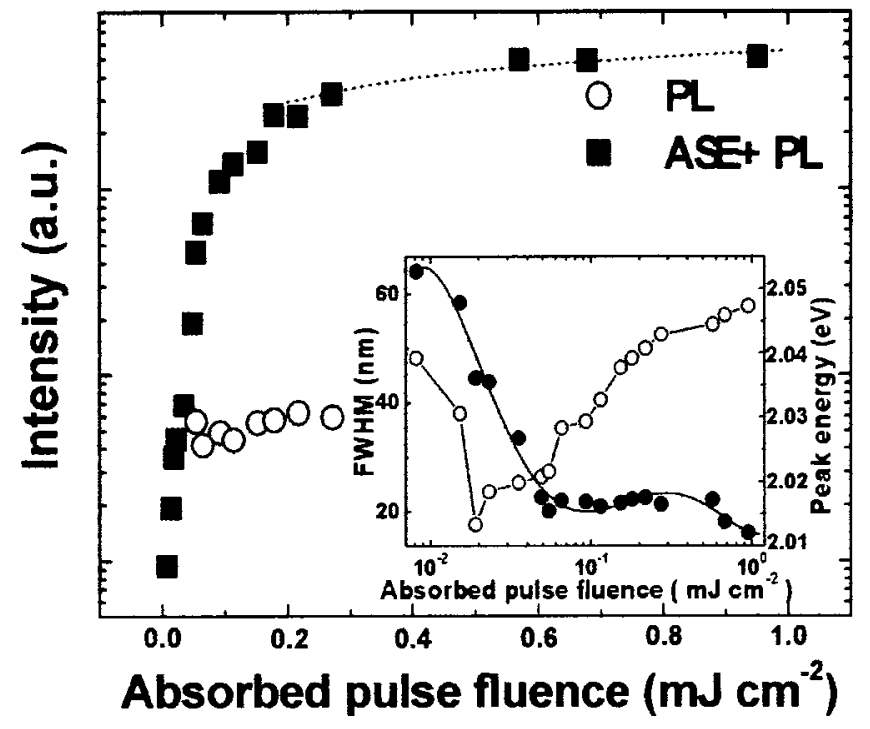

FIG. 3. Dependence of the total integrated emitted intensity (ASE+PL) and of the PL on the pump energy density. The dotted line is the best fit of the data between 0.22 and $0.95 \mathrm{~mJ} \mathrm{~cm}^{-2}$ to the square-root behavior predicted by the bimolecular annihilation model. Inset: emission linewidth (FWHM, full circles) and peak energy (open circles) as a function of pump pulse fluence. The continuous lines are guides for the eye.

excitation density (inset of Fig. 3). The redshift can be attributed to the rise of ASE (with a peak $2.013 \mathrm{eV}$ ) on the spontaneous emission background (peaked at $2.039 \mathrm{eV}$ ). ${ }^{21}$ The blueshift indicates the competition between stimulated emission and energy relaxation: Higher excitation density implies faster depletion by ASE, before relaxation occurs. A similar blueshift at a higher excitation density has been previously observed in polymers and attributed to the competition between gain and the excitation diffusion on the longer chains. ${ }^{22}$ In this case, relaxation might be due to both interchain migration within a distribution of site energies (conformers, local arrangements, $X$ traps, etc.) and intrachain relaxation (IC). We point out that, as the relaxation by IC in oligothiophenes usually takes place in the first few hundreds of femtosecond after the excitation, ${ }^{23}$ the observed blueshift of the gain is a signature of a very high gain rate. Indeed, a gain cross section $\sigma_{g}$ of $6 \times 10^{-16} \mathrm{~cm}^{2}$ has been estimated from a pump-probe measurements at $610 \mathrm{~nm}$. This result underestimates the actual gain cross section, as a unit quantum efficiency for the emitting species is assumed in the calculation. Yet, this value is two orders of magnitude larger than in PPV oligomers ${ }^{24}$ and methyl-substituted PPV, ${ }^{7}$ one order of magnitude larger than $\sigma_{g}$ in methyl-substituted ladder-type polymers ${ }^{6}$ and comparable to that of polythiophenes films. ${ }^{25}$

From these results, it is clear that $\mathrm{T} 5 \mathrm{oCx}$ is an appealing candidate for organic laser devices. Actually, laser emission was obtained by optical pumping of a neat film of $\mathrm{T} 5 \mathrm{oCx}$, using the plane-concave resonator previously described (inset in Fig. 4). We chose this resonator because of its stability and of the better mode matching between the excitation mode and the cavity mode, as compared to a plane-parallel resonator. The input-output characteristic of the device, shown in Fig. 4, shows a clear threshold corresponding to an excitation density as low as $18 \mu \mathrm{J} / \mathrm{cm}^{2}$, followed by a linear increase, as expected for laser action. The efficiency of the 


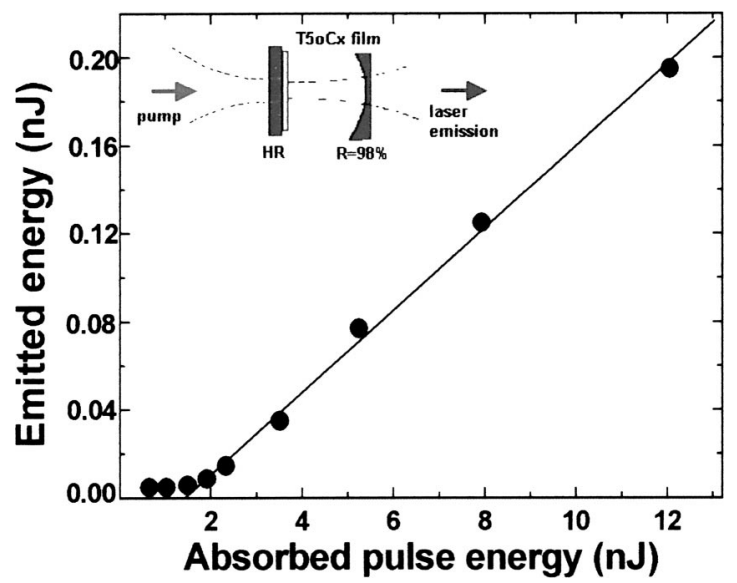

FIG. 4. Laser emission energy vs pump energy. The solid line is the best linear fit to the experimental data. Inset: geometry of the laser cavity.

laser was estimated by a numerical linear fit of the characteristic above threshold to be $1.9 \%$, which is among the highest so far reported for organic lasers. The laser mode has a $\mathrm{TEM}_{00}$ beam quality with an almost diffraction-limited divergence, with a full width at half maximum (FWHM) of the laser line limited by the resolution of the spectrometer $(1.2$ $\mathrm{nm})$. We emphasize that this laser produces a single longitudinal mode emission with a stable transverse cavity mode. Finally, we were able to tune the laser emission wavelength by changing the cavity length. Single-mode laser emission, with almost constant output energy, was indeed tuned in a wavelength range as wide as $37 \mathrm{~nm}$ centered around $594 \mathrm{~nm}$, from the yellow $(575 \mathrm{~nm})$ to the red $(612 \mathrm{~nm}$, Fig. 5). Though not common in organic lasers, such a wide tunability is typical of our functionalized oligothiophenes, ${ }^{18}$ due to their broad spectral region of gain. ${ }^{17}$ For the device spectra of Fig. 5, the resonator length was $\sim 6 \mu \mathrm{m}$, and the cavity internal losses per pass were $<10 \%,{ }^{26}$ probably due to film scattering or nonperfect alignment of the cavity.

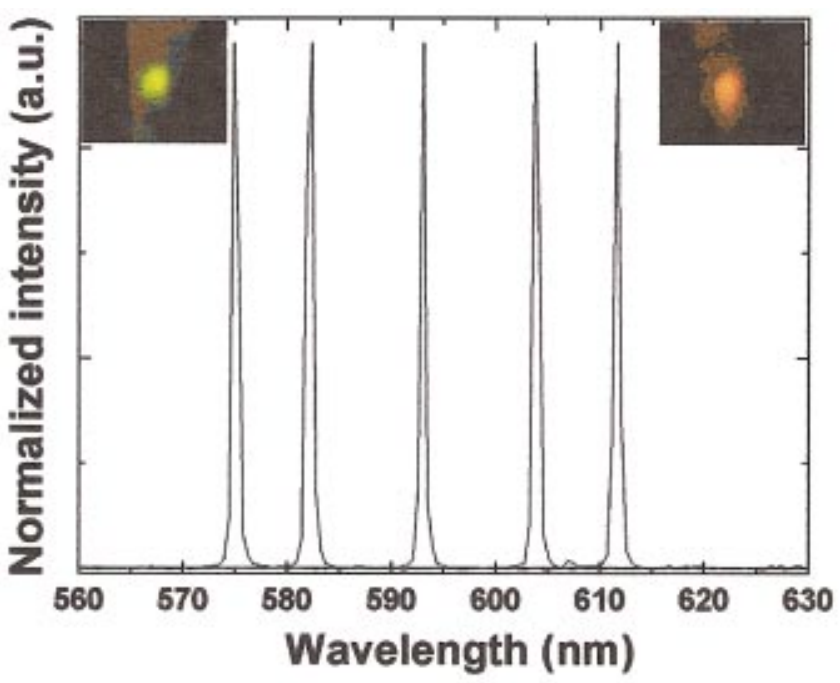

FIG. 5. (Color) Normalized emission spectra of the T5oCx laser obtained at different cavity lengths, showing $\mathrm{TEM}_{00}$ emission from the yellow to the red (photographed laser spots in the insets).
In conclusion, the very low threshold for line narrowing $\left(20 \mu \mathrm{J} \mathrm{cm}^{-2}\right)$, the large value of the cross section $\sigma_{g}(6$ $\times 10^{-16} \mathrm{~cm}^{2}$ ), together with the excellent processability and film-forming capability, suggest that thienyl-S,S-dioxide oligomers can compete with conjugated polymers in the realization of organic-based semiconductor lasers. In the present work, we have indeed realized a single-mode laser exhibiting figures of merit (threshold, efficiency, tunability, and beam quality) which are among the best so far reported for organic lasers.

${ }^{1}$ D. Moses, Appl. Phys. Lett. 60, 3215 (1992).

${ }^{2}$ N. Tessler, G. J. Denton, and R. H. Friend, Nature (London) 382, 695 (1996).

${ }^{3}$ W. Graupner, G. Leising, G. Lanzani, M. Nisoli, S. De Silvestri, and U. Scherf, Phys. Rev. Lett. 76, 847 (1996).

${ }^{4}$ S. V. Frolov, W. Gellerman, M. Ozaki, K. Yoshino, and Z. V. Vardeny, Phys. Rev. Lett. 78, 729 (1997).

${ }^{5}$ G. J. Denton, N. Tessler, M. A. Stevens, and R. H. Friend, Adv. Mater. 9, 547 (1997).

${ }^{6}$ S. Stagira, M. Nisoli, G. Cerullo, M. Zavelani-Rossi, S. De Silvestri, G. Lanzani, W. Graupner, and G. Leising, Chem. Phys. Lett. 289, 205 (1998).

${ }^{7}$ B. Schweitzer, G. Wengmann, H. Giessen, D. Hertel, H. Bassler, and R. F. Mahrt, Appl. Phys. Lett. 72, 2933 (1998).

${ }^{8}$ S. V. Frolov, M. Shkunov, Z. V. Vardeny, and K. Yoshino, Phys. Rev. B 56, 4363 (1997).

${ }^{9}$ V. G. Kozlov, V. Bulović, P. E. Burrows, and S. R. Forrest, Nature (London) 389, 362 (1997).

${ }^{10}$ M. Bergreen, A. Dodabalapur, R. E. Slusher, and Z. Bao, Nature (London) 389, 466 (1997)

${ }^{11}$ M. Bergreen, A. Dodabalapur, and R. E. Slusher, Appl. Phys. Lett. 71, 2230 (1997)

${ }^{12}$ S. Stagira, M. Zavelani-Rossi, M. Nisoli, S. De Silvestri, G. Lanzani, C. Zenz, P. Mataloni, and G. Leising, Appl. Phys. Lett. 73, 2860 (1998).

${ }^{13}$ M. D. McGehee, M. A. Diaz-García, F. Hide, R. Gupta, E. K. Miller, D. Moses, and A. J. Heeger, Appl. Phys. Lett. 72, 1536 (1998).

${ }^{14}$ M. Anni, G. Gigli, V. Paladini, R. Cingolani, G. Barbarella, L. Favaretto, G. Sotgiu, and M. Zambianchi, Appl. Phys. Lett. 77, 2458 (2000).

${ }^{15}$ G. Gigli, O. Inganas, M. Anni, M. De Vittorio, R. Cingolani, G. Barbarella, and L. Favaretto, Appl. Phys. Lett. 78, 1493 (2001).

${ }^{16}$ G. Barbarella, L. Favaretto, M. Zambianchi, O. Pudova, C. Arbizzani, A. Bongini, and M. Mastragostino, Adv. Mater. 10, 551 (1998).

${ }^{17}$ M. Anni, G. Gigli, R. Cingolani, M. Zavelani-Rossi, C. Gadermaier, G. Lanzani, G. Barbarella, and L. Favaretto, Appl. Phys. Lett. 78, 2679 (2001).

${ }^{18}$ M. Zavelani-Rossi, G. Lanzani, S. De Silvestri, M. Anni, G. Gigli, R. Cingolani, G. Barbarella, and L. Favaretto, Appl. Phys. Lett. 79, 4082 (2001).

${ }^{19}$ F. Garnier, G. Horowitz, P. Valat, F. Kouki, and V. Wintgens, Appl. Phys. Lett. 72, 2087 (1998).

${ }^{20}$ The sublinear evolution of the integrated intensity on the pump is observed for high pump power $\left(>0.2 \mathrm{~mJ} \mathrm{~cm}^{-2}\right)$ indicates the presence of nonradiative decay processes, increasing as the pump energy is increased. The resulting gain saturation has been mainly attributed to nonlinear effects, like bimolecular exciton annihilation, induced by the high density of excitons formed at very intense photon fluxes. This behavior is confirmed by a square-root dependence of the high-power emission on excitation density, consistent with the bimolecular annihilation model (dotted fitting curve in Fig. 3).

${ }^{21}$ The ASE peak position depends on both the maximum gain energy and on the maximum transmission energy of the waveguides, depending on the film thickness.

${ }^{22}$ E. M. Conwell, in Organic Electronic Materials, edited by R. Farchioni and G. Grosso (Springer, Berlin, 2001), pp. 170-174.

${ }^{23}$ S. V. Frolov, C. Kloc, B. Batlogg, M. Wohlgenannt, X. Jiang, and Z. V. Vardeny, Phys. Rev. B 63, 205203 (2001).

${ }^{24}$ E. S. Maniloff, V. I. Klimov, and D. W. McBranch, Phys. Rev. B 56, 1876 (1997).

${ }^{25}$ A. Ruseckas, M. Theander, M. R. Andersson, M. Svensson, M. Prato, O. Inganäs, and V. Sundström, Chem. Phys. Lett. 322, 136 (2000).

${ }^{26}$ We obtained such information from the spectral mode spacing of the modulated PL recorded when pumping below threshold and from the FWHM of the laser line (Fig. 5), respectively. 